\title{
Clinical Trial of a Feline Pheromone Analogue for Feline Urine Marking
}

\author{
Niwako OGATA ${ }^{1)}$ and Yukari TAKEUCHI ${ }^{2}$ \\ 1) Veterinary Behavior Clinic FAU, 3-34-7 Yamadanishi, Suita-shi, Osaka 565-0824 and ${ }^{2)}$ Laboratory of Veterinary Ethology, The \\ University of Tokyo, 1-1-1 Yayoi, Bunkyo-ku, Tokyo 113-8657, Japan
}

(Received 26 June 2000/Accepted 29 October 2000)

ABSTRACT. Thirty-six cases of feline urine marking problem were collected through the cooperation of veterinary practitioners in the Kanto, Chubu, and Kansai areas in Japan, for an assessment of the clinical effect of treatment with a synthetic analogue of a feline cheek gland pheromone-like product. The mean frequency of urine marking was 14.2 times/week (median, 10; range, 1-77) at pre-treatment week (preW), and decreased significantly from the first week of treatment, dropping to 4.2 times/week (median, 2; range, $0-44$ ) at the fourth week of treatment. This effect continued until the fourth week after cessation of treatment. These 36 cases were divided into 3 groups based on the effectiveness of treatment as demonstrated in the fourth week of treatment; $37 \%$ was categorized as the totally eliminated group (urine marking was not seen), $40 \%$ as the reduced group (the frequency of urine marking was equal to or less than $50 \%$ that of the preW), and $23 \%$ as the unchanged group (the frequency of urine marking was more than $50 \%$ that of the preW). Effectiveness of treatment in these groups was $38 \%, 24 \%$, and $38 \%$ at the fourth week after the cessation of treatment, respectively. The decreasing rate of urine marking was compared between cats with and without intercat aggression, and it was revealed that the frequency of marking was sustained at high level in cats with intercat aggression. These results suggest that this pheromone treatment is as effective in Japan as has been reported in other countries for solving feline urine marking problems.

KEY WORDS: feline, intercat aggression, pheromone treatment, spraying, urine marking.

J. Vet. Med. Sci. 63(2): 157-161, 2001

The elimination problem is reported to be the most common among feline behavioral problems in Western countries $[1,9]$, though no comparable data is available in Japan. This elimination problem is usually categorized as inappropriate elimination or as urine marking, which occur independent of each other. Generally, urine marking is thought to be a problem of intact male cats, though this problem may occur in females and in neutered animals. The rate of incidence is much higher in males than in females, and even in cats neutered between six and ten months old, $12 \%$ of males will still start urine marking afterward, as compared with $4 \%$ of females $[5,13]$.

Recently, it has been suggested that urine marking is not simply the result of a territorial instinct or a sexual motivation, but an expression of 'passive aggression' or 'anxiety' [11]. Subsequently, veterinary clinicians might treat this problem through environmental management, behavior modification, and pharmacological invention, all of which should be considered for each case $[10,13]$. In addition to these treatments, the pheromone treatment, a new approach in companion animals, was introduced to Japan in 1998. The product (Feliway ${ }^{\mathrm{TM}}$, Virbac, Japan) used in this treatment is reported to be derived from a synthetic feline facial pheromone that has a calming effect on cats, and has been found to inhibit the frequency of urine marking if it is applied to the immediate environment $[4,8,12]$.

However, the effects of this pheromone treatment differed among countries, i.e. the 'success rate' ranged between 74 and $97 \%[4,8,12]$. Although the reason for this difference is unclear, various factors such as a trial design, background of cats, environmental inconsistency, and cultural differences would have affected the trial outcome.
Even if it may be difficult to identify the actual reason(s), it would be important for veterinary clinicians to know the practical value of the new treatment under their particular circumstances. In this study, we therefore undertook a survey to examine the effect of this pheromone treatment on urine marking problem in cats living in households in Japan and compared the efficacy with those reported in other countries.

\section{MATERIALS AND METHODS}

Case collection: Cases were collected through the cooperation of 52 veterinary hospitals in the Kansai, Kanto, Chubu, and Hokkaido areas in Japan. The cases used in this study were selected by veterinary practitioners after careful examination. Concretely, we excluded cases in which any physical cause might have been considered as the source of the problems; cases of very young animals ( $<6$ months old); and cases in which the owner could not definitively identify the marking cat in a multiple cat household or the urine marking occurred only outdoors. Cases in which the problem had already been treated by other methods, especially with drugs such as benzodiazepines or antidepressants, were also excluded from this study. In order to reduce ambiguity and make an exact behavioral diagnosis, all cases were limited to those in which the cats marked on vertical surfaces. Through this process of selection, 55 cases seen between November 1998 through March 1999 were selected and used in this study.

Instead of conducting a face-to-face interview, we asked the owners to fill out a questionnaire by the respective veterinarians, which provided complete and extensive informa- 
tion on the cat at the beginning of the trial. The information requested included a profile of the cat, a history of the problems, the presence of other animals in the same household, and the presence of intercat aggression.

Trial design: The design of the study followed that of Frank et al. [4]. The observation period was divided into three parts: (1) pre-treatment period (1 week), (2) pheromone application period (4 weeks), (3) post-treatment period (4 weeks). No other treatments such as behavior modification, environmental modification and/or drug administration than pheromone application were conducted throughout the trial period.

Pre-treatment period: The owners were instructed to fill out a control data sheet to determine the daily frequency of urine marking for one week just prior to the pheromone treatment (pre week; preW).

Pheromone application period: The treatment consisted of application of the synthetic pheromone (Feliway ${ }^{\mathrm{TM}}$, Virbac, Japan) per the manufacturer's instructions; the pheromone was applied to all urine marks as well as to other locations that the cat might find attractive. The pheromone product was to be applied once daily (two to three times daily in case of a multiple cat household) for a period of 4 weeks (weeks 1 to 4; W1 to W4). The owners were asked to make a daily recording of any urine marks found during this period.

Post-treatment period: After 4 weeks of treatment, owners were asked to stop applying the treatment and to record the frequency of urine marking for the following 4 weeks (week 5 to 8 ; W5 to W8).

After the trial period had ended, the owners were asked to give their impressions about the change of the relationship with their cat in order that we could gauge their satisfaction.

Statistical analysis: The statistical analysis was performed by Wilcoxon's signed rank test with Ryan's procedure for comparison of the frequency of urine marking between preW and each subsequent week (W1 to W8). Chisquare test was performed for comparison of treatment effects between W4 and W8 after the prognoses had been categorized into the reduced group (the frequency of urine marking was equal to or less than $50 \%$ that of the preW) or the unchanged group (the frequency of urine marking was more than $50 \%$ that of the preW). In order to examine the difference of treatment effect on urine marking relative to sexual status or in with/without intercat aggression groups, one-way or two-way ANOVA with repeated-measures was used. The significance level for all statistical tests was set at 0.05 .

\section{RESULTS}

Nineteen cats were dropped from the study because of incomplete records of observation. The study was completed for a total of 36 cats. As shown in Table 1, subjects included 6 intact males, 19 castrated males, 5 intact females, and 6 ovariohysterectomized females. Twenty-eight cats belonged to 28 respective multiple cat households ( 2 to 15 cats in a household; mean 4.8 , median 4 ) and 8 cats belonged to 8 single cat households. The age of cats ranged from 8 months to 15 years old (mean 5.1 years, median 5 years). Five out of 36 cats were outdoors from one to nine hours a day, and 2 cats were in a cage for about $12 \mathrm{hr}$. Others were kept totally indoors, $24 \mathrm{hr}$ a day. Duration of the problem ranged among all cases from 1 month to 10.5 years (mean 27.6 months, median 13.5 months). In multiple cat households, duration of the problem ranged from 3 months to 10.5 years (mean 30.9 months, median 17 months), and ranged from 1 month to 5.2 years (mean 16 months, median 2 months) in single cat households.

The actual number of urine markings of each individual cat found weekly during the pre-treatment, treatment, and post-treatment periods are shown in Table 1. The urine markings of most animals gradually decreased during the treatment period and were maintained even after treatment had been stopped. Wilcoxon's signed rank test with Ryan's procedure revealed a significant difference between preW and each week (W1 to W8). The mean reduction ratios of urine marking compared to preW were $68.5 \%$ (W4, last week of treatment) and $64.6 \%$ (W8, 4 weeks after cessation of treatment).

Figure 1 shows the decreasing time course of urine marking categorized into 4 groups by sexual status. Though the tendency toward decreasing of the mean was similar among groups, the level of efficacy differed depending on sexual status. The male group showed a more dramatic decrease than that in the female group, and both castrated and spayed groups showed a comparatively mild decrease, though the difference among groups was not significant.

All cases were divided into 3 groups based on the effectiveness of the treatment, namely the totally eliminated group, the reduced group, and the unchanged group. The ratio of each group at $\mathrm{W} 4$ or $\mathrm{W} 8$ is shown in Fig. 2. Although the number of animals decreased due to lack of data, the urine markings in $37.1 \%$ of cases at the last week of treatment period (W4) and in $37.9 \%$ at the last week of the post-treatment period (W8) were completely vanished. When the total number of cases in the totally eliminated and reduced groups (27 cases, $77.1 \%$ at $\mathrm{W} 4 ; 18$ cases, $62.1 \%$ at W8) and that in the unchanged group ( 8 cases, $22.9 \%$ at W4; 11 cases, $37.9 \%$ at W8) were compared by chi-square test, there was no statistical difference between W4 and W8 though the success ratio (consisting of the totally eliminated and reduced groups) seemed to be decreased at $\mathrm{W} 8$, as can be seen in Fig. 2.

Fifteen out of 28 multiple cats households showed intercat aggression (see Table 1). The decreasing profiles of urine marking in cases with $(n=15)$ and without $(n=13)$ intercat aggression, respectively, are shown in Fig. 3. Although the frequency of urine marking in the cases with intercat aggression was drastically decreased by the treatment, it sustained a high level, which caused a significant difference between groups $(\mathrm{p}<0.05)$.

The owners' impressions about the changes of the relationship with their cats are also shown in Table 1. Fifteen 
Table 1. The number of urine markings found weekly during pre-treatment, treatment and post-treatment weeks

\begin{tabular}{|c|c|c|c|c|c|c|c|c|c|c|c|c|c|c|c|c|c|}
\hline No. & Sexual status & Household ${ }^{\text {a) }}$ & $\operatorname{Age}(\mathrm{Y})$ & Breed $^{\text {b) }}$ Hist & istory $\left.(\mathrm{M})^{\mathrm{c}}\right)$ & Total no. ${ }^{\text {d) }}$ & PreW & $\mathrm{W} 1$ & W2 & W3 & W4 & W5 & W6 & w7 & W8 & Aggression & Relation $^{\text {e) }}$ \\
\hline 1 & Male & Multi & 3 & $\mathrm{NFC}$ & 36 & 4 & 7 & 0 & 0 & 0 & 0 & 0 & 1 & 3 & 5 & & Better \\
\hline 2 & Male & Multi & 6 & DSH & 60 & 4 & 12 & 10 & 9 & 9 & 9 & 9 & 9 & 9 & 9 & & Unchanged \\
\hline 3 & Male & Multi & 2 & DSH & 13 & 9 & 77 & 6 & 19 & 25 & 44 & 24 & 33 & 37 & ND & + & Unchanged \\
\hline 4 & Male & Single & 1 & Munchkin & 2 & 1 & 15 & 11 & 4 & 2 & 0 & 0 & 1 & 2 & 0 & & Better \\
\hline 5 & Male & Single & 1 & Munchkin & 1 & 1 & 31 & 22 & 18 & 14 & 10 & 10 & ND & ND & ND & & Better \\
\hline 6 & Male & Single & 1 & Munchkin & 2 & 1 & 14 & 13 & 8 & 4 & 1 & 0 & 0 & 0 & 0 & & Better \\
\hline 7 & Castrated male & Multi & 6 & DSH & 7 & 6 & 2 & 1 & 2 & 2 & 0 & 0 & 0 & 1 & 0 & & Better \\
\hline 8 & Castrated male & Multi & 6 & DSH & 9 & 7 & 63 & 8 & 8 & 3 & 5 & 6 & 8 & 10 & 6 & + & Unchanged \\
\hline 9 & Castrated male & Multi & 5 & DSH & 13 & 2 & 10 & 7 & 4 & 4 & 3 & 3 & 4 & 3 & 3 & + & Others \\
\hline 10 & Castrated male & Multi & 3 & DSH & 13 & 5 & 16 & 14 & 8 & 8 & 6 & 5 & 9 & 9 & 11 & + & Others \\
\hline 11 & Castrated male & Multi & 8 & $\mathrm{ASH}$ & 31 & 2 & 6 & 6 & 3 & 2 & 2 & 2 & 1 & 2 & 3 & & Others \\
\hline 12 & Castrated male & Multi & 7.5 & DSH & 36 & 2 & 1 & 1 & 0 & 0 & 0 & 0 & ND & ND & ND & + & Unchanged \\
\hline 13 & Castrated male & Multi & 8 & DSH & 56 & 4 & 18 & 9 & 5 & 2 & 8 & 9 & 7 & 7 & 16 & + & Unchanged \\
\hline 14 & Castrated male & Multi & 7 & DSH & 65 & 9 & 9 & 3 & 1 & 0 & 0 & 0 & 0 & 0 & 0 & + & Better \\
\hline 15 & Castrated male & Multi & 15 & DSH & 126 & 4 & 1 & 1 & 0 & 0 & 0 & ND & ND & ND & 0 & & Unchanged \\
\hline 16 & Castrated male & Multi & 3.5 & DSH & 12 & 15 & 9 & 7 & 5 & 7 & 4 & 5 & 6 & 5 & 6 & + & Unchanged \\
\hline 17 & Castrated male & Multi & 12 & DSH & 12 & 3 & 9 & 7 & 7 & 6 & 6 & 5 & ND & ND & ND & + & Unchanged \\
\hline 18 & Castrated male & Multi & 6 & DSH & 48 & 2 & 30 & 12 & 16 & 13 & ND & 15 & ND & ND & ND & + & Unchanged \\
\hline 19 & Castrated male & Multi & 9 & DSH & 72 & 5 & 11 & 8 & 9 & 7 & 8 & ND & ND & ND & 10 & + & Unchanged \\
\hline 20 & Castrated male & Multi & 4 & DSH & 14 & 2 & 13 & 14 & 14 & 10 & 9 & 9 & 10 & 13 & 14 & + & Unchanged \\
\hline 21 & Castrated male & Multi & 3 & DSH & 6 & 8 & 7 & 2 & 4 & 0 & 2 & 1 & 1 & 0 & 0 & + & Unchanged \\
\hline 22 & Castrated male & e Multi & $>1$ & DSH & 7 & 2 & 28 & 2 & 0 & 1 & 0 & 11 & 5 & 3 & 2 & & Better \\
\hline 23 & Castrated male & Single & 6 & DSH & 28 & 1 & 4 & 2 & 2 & 0 & 0 & 4 & 3 & 3 & ND & & Better \\
\hline 24 & Castrated male & Single & 1 & ASH & 1 & 1 & 10 & 10 & 2 & 3 & 2 & 4 & 8 & 5 & 7 & & Unchanged \\
\hline 25 & Castrated male & Single & 5 & DSH & 31 & 1 & 11 & 4 & 1 & 0 & 0 & 0 & 0 & 0 & 0 & & Better \\
\hline 26 & Female & Multi & 0.7 & DSH & 3 & 3 & 3 & 0 & 0 & 0 & 0 & 0 & 0 & 0 & 0 & & Better \\
\hline 27 & Female & Multi & 3 & NFC & 35 & 4 & 7 & 0 & 0 & 0 & 0 & 0 & 3 & 3 & 5 & & Better \\
\hline 28 & Female & Multi & 3 & DSH & 20 & 4 & 8 & 9 & 4 & 2 & 1 & 1 & 1 & 2 & 0 & & Others \\
\hline 29 & Female & Multi & 2 & Persian & 10 & 3 & 6 & 6 & 3 & 2 & 1 & 1 & 1 & 3 & 4 & & Better \\
\hline 30 & Female & Single & 1 & Munchkin & 1 & 1 & 12 & 12 & 6 & 2 & 0 & 0 & 0 & 0 & 0 & & Better \\
\hline 31 & Spayed female & Multi & 4 & DSH & 10 & 2 & 4 & 5 & 2 & 4 & 4 & ND & ND & ND & ND & & Unchnaged \\
\hline 32 & Spayed female & Multi & 8 & DSH & 36 & 15 & 13 & 10 & 9 & 7 & 11 & 8 & 9 & 9 & 11 & + & Unchnaged \\
\hline 33 & Spayed female & Multi & 5 & Siamese & 36 & 2 & 1 & 0 & 2 & 1 & 2 & 3 & 1 & 0 & 0 & + & Unchanged \\
\hline 34 & Spayed female & Multi & 8 & DSH & 72 & 4 & 3 & 3 & 2 & 2 & 1 & 2 & 2 & 1 & 1 & & Unchanged \\
\hline 35 & Spayed female & Multi & 3 & DSH & 7 & 3 & 17 & 14 & 10 & 7 & 7 & 3 & 4 & 4 & 3 & & Better \\
\hline 36 & Spayed female & Single & 11 & DSH & 62 & 1 & 22 & 0 & 0 & 0 & 0 & 3 & 7 & 10 & 6 & & Better \\
\hline \multicolumn{6}{|c|}{ Mean } & & 4.17 & 6.64 & 5.19 & 4.14 & 4.17 & 4.33 & 4.62 & 4.97 & 4.21 & & \\
\hline \multicolumn{6}{|c|}{ Median } & & 10.0 & 6.5 & 4.0 & 2.0 & 2.0 & 3.0 & 3.0 & 3.0 & 3.0 & & \\
\hline \multicolumn{6}{|c|}{ Mean reduction rate $(\%)$} & & 0.003 & 8.46 & 54.82 & 66.27 & 68.546 & 63.84 & 65.81 & 66.15 & 64.62 & & \\
\hline
\end{tabular}

ND; no data.

a) Multi: multiple cat household, Single: single cat household.

b) NFC: Norway forest cat, ASH: American shorthair, DSH: Domestic shorthair.

c) History: the length of the problem.

d) Total number of cats including the problem cat in the household.

e) Owner's impression about the change of relationship with the cat after pheromone treatment.

out of 36 owners $(41.7 \%)$ felt that the relationship became better; 17 owners $(47.2 \%)$ thought that nothing was changed regarding the relationship; and 4 owners $(11.1 \%)$ considered that the treatment had undesirable side effects, such as making their animals more aggressive or unfriendly than before (Table 1, Relation ${ }^{\mathrm{e})}$; Others).

\section{DISCUSSION}

In the present study, it was shown that the synthetic pher- omone product decreased the frequency of urine marking by cats in households in Japan. This result is consistent with findings in previous reports $[4,8,12,15]$, in which researchers defined the success rate as the ratio of the animals whose markings at the last week of treatment were reduced compared to their markings at the preW. The success rate among our subjects calculated by this method was $91.6 \%$ (33/36 animals), which is higher than the $74 \%$ success rate found in a USA study [4] but similar to that of $97 \%$ found in a French study [12] and that of $91 \%$ found in a UK study 


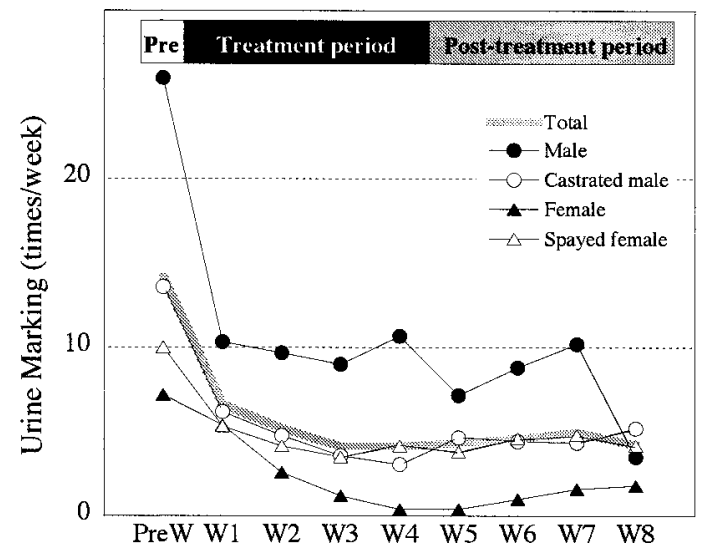

Fig. 1. Decreasing profiles of urine marking throughout pheromone treatment trial in cases categorized into 4 groups by sexual status.

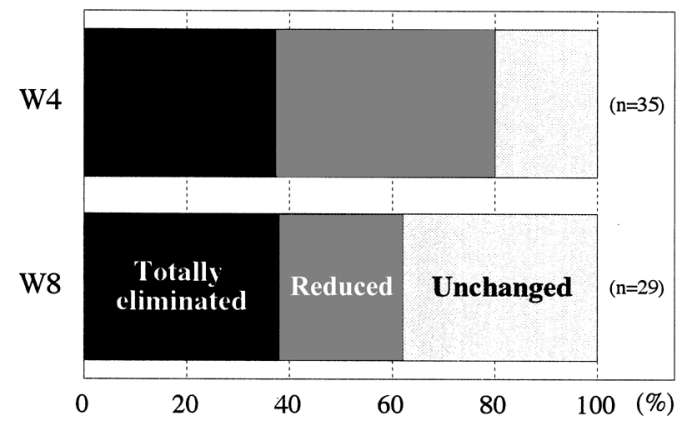

Fig. 2. The effect of pheromone treatment evaluated at the last week of pheromone application (W4) and at the last week of the posttreatment period (W8). See details in the text. Totally eliminated, urine marking was not seen during the week; Reduced, the frequency of urine marking was equal to or less than $50 \%$ that of the preW; Unchanged, the frequency of urine marking was more than $50 \%$ that of the preW.

[15]. The differences in findings among these studies, in which the experimental designs were very similar, might be caused not only by regional differences but by differences among environmental conditions such as the total number of cats in a household, or various differences in the animals' backgrounds relative to such factors as age, duration of the problem, and/or the presence of other behavioral problems. However, the definition of the term 'success rate' used in these studies may require clarification; we found that in some animals the number of markings showed only a subtle fluctuation (see results for animal numbers 2, 17, 19, 20, and 32, as shown in Table 1). In this study, we used this term to indicate the percentage of animals whose number of markings was reduced to equal to or less than $50 \%$ that of the number of markings of preW. The value $77.1 \%$ is thought to be more reliable and objective in terms of the real effect of treatment.

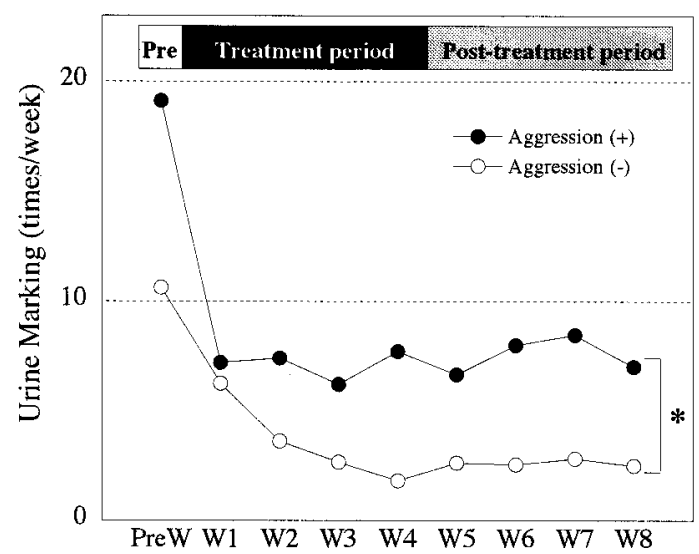

Fig. 3. The difference of the treatment effect on urine marking between cats with intercat aggression and cats without intercat aggression. *, $\mathrm{p}<0.05$ by ANOVA.

Although worldwide studies including the present study were not designed to compare the effects of a placebo with that of the pheromone product, that sort of comparison is imminently necessary in order to overcome the varied trial condition such as the existence of estrous female around the problem cat and/or owner's miscounting of marking spots [7]. Notably, in this study, the success rate of pheromone treatment $(77.1 \%)$ was relatively higher than that of pharmacological treatment $(55-75 \%)$ [13]. When statistically compared, no evidence of relapse at the last week of the post-treatment period (W4) was seen among our subjects (Fig. 2). Relapse with pharmacological treatment has been reported to be $90 \%$ with benzodiazepine [2], $50 \%$ with buspirone $[2,6]$, and $75 \%$ with clomiplamine [3]. These findings, taken together with the fact that no drug has been registered for feline behavioral problems in Japan and further, that administering a drug to a cat is difficult for the pet owner, pheromone treatment seems preferable to drug treatment in Japan.

In this study, no difference was observed in the duration of the problem relative to multiple cat households (data not shown), which is in accord with the findings in previous reports $[8,15]$. On the other hand, we found that the frequency of marking by cats with intercat aggression showed a significantly smaller decrease in response to treatment. However, the fact that the comparison of normalizing data (the frequency of urine marking at preW was set as the standard level) could not detect this difference leads us to speculate that the difference between aggressive and nonaggressive cats may be derived from the difference between urine markings at the preW. Taking these findings together, urine marking problems in cats might be more affected by environmental factors than by learning factors. Therefore, it may be useful, in future assessment of the efficacy of the pheromone treatment, to place more emphasis on environmental factors and the presence of other behavior problems as potential causes of urine marking. 
It is interesting to note that all the cats, whose owners felt that the relationship with their cat became better, were categorized into 'reduced group' at the fourth week of pheromone application. This fact may indicate that some owners who have cats with this kind of problem may be able to reconstruct better relationship with their cats if the frequency of urine marking can be reduced. Although the impression of the owner is not a scientific parameter, this factor will exert great influence on the individual animal's future, especially in case of treatment for behavioral problems [14]. Therefore, veterinary clinicians should treat not only medical problems but also behavioral problems in order to help owners have better relationship with their companion animals.

ACKNOWLEDGMENTS. The authors are grateful to Drs. Tomiya Uchino, Norio Kogure, Makoto Tatematsu, Makiko Awazu, Kuninobu Toda, Kiyoshi Matsushita, and the other veterinarians in the 52 veterinary hospitals for their help in collecting data from pet owners. They also acknowledge Ceva Sante Animale and Virbac in Japan for generous supply of their products for this study.

\section{REFERENCES}

1. Borchelt, P. L. and Voith, V. L. 1996. Elimination behavior problems in cats. pp. 179-190. In: Readings in Companion Animal Behavior (Voith, V. L. and Borchelt, P. L. eds.), Veterinary Learning Systems, Trenton.

2. Cooper, L. and Hart, B. L. 1992. Comparison of diazepam with progestin for effectiveness in suppression of urine spraying behavior in cats. J. Am. Vet. Med. Assoc. 200: 797-801.

3. Dehasse, J. 1997. Feline urine spraying. Appl. Anim. Behav. Sci. 52: 365-371

4. Frank, D., Erb, H. N. and Houpt, K. A. 1999. Urine spraying in cats: presence of concurrent disease and effects of a pheromone treatment. Appl. Anim. Behav. Sci. 61: 263-272.
5. Hart, B. L. and Cooper, L. L. 1984. Factors relating to urine spraying and fighting in prepubertally gonadectomized cats. $J$. Am. Vet. Med. Assoc. 184: 1255-1258.

6. Hart, B. L., Eckstein, R. A., Powell, K. L. and Dodman, N. H. 1993. Effectiveness of buspirone on urine spraying and inappropriate urination in cats. J. Am. Vet. Med. Assoc. 203: 254258.

7. Hart, B. L. and Cliff, K. D. 1996. Interpreting published results of extra-label drug use with special reference to reports of drugs used to correct problem behavior in animals. J. Am. Vet. Med. Assoc. 209: 1382-1385.

8. Hunthausen, W. 2000. Evaluating a feline facial pheromone analogue to control urine spraying. Vet. Med. 95: 151-155.

9. Kerby, G. and Macdonald, D. W. 1988. Cat society and the consequences of colony size. pp. 67-81. In: The Domestic Cat: The Biology of Its Behaviour (Turner, D. C. and Bateson, P. eds.), Cambridge University Press, Cambridge.

10. Lansberg, G., Hunthausen, W. and Ackerman, L. 1997. Handbook of Behavior Problems of the Dog and Cat. Butterworth Heinemann, Oxford.

11. Overall, K. L. 1998. Tracing the roots of feline elimination disorders to aggression. Vet. Med. 93: 363-366.

12. Pageat, P. 1996. Functions and use of the facial pheromones in the treatment of urine marking in the cat; interest of a structural analogue. pp. 197-198. In: Proceedings and Abstracts of the XXI Congress of the World Small Animal Vet. Assoc. (Johnson, D. and Waner, T. eds.)

13. Simpson, B. S. 1998. Feline Housesoiling. Part II. Urine and Fecal Marking. Compend. Contin. Educ. Pract. Vet. 20: 13311339.

14. Takeuchi, Y., Houpt, K. A. and Scarlett, J. M. 2000. Evaluation of treatments for separation anxiety in dogs. J. Am. Vet. Med. Assoc. 217: 342-345.

15. White, J. C. and Mills, D. S. 1997. Efficacy of synthetic facial pheromone (F3) analogue (Feliway) for the treatment of chronic non-sexual urine spraying by the domestic cat. p. 262. In: Proceedings of the First Conference on Veterinary Behavioural Medicine. Universities Federation for Animal Welfare, UK. 